\title{
INITIAL INVESTIGATION INTO REAL 3D BODY SCANNING VERSUS AVATARS FOR THE VIRTUAL FITTING OF GARMENTS
}

\author{
Monika Balach ${ }^{1, *}$, Agnieszka Cichocka ${ }^{1}$, Iwona Frydrych ${ }^{1}$, Marc Kinsella $^{2}$ \\ 1 Technical University of Lodz, Faculty of Material Technologies and Textile Design, Institute of Architecture of Textile, Lodz 90-924, \\ 116 Zeromskiego Street, Poland \\ 2 TETRAD, Hartford Mill, Swan St., Preston PR1 5 PQ, England \\ *Correspondence to: monikabalach@mail.com; Tel. +(48) (42) 6365522
}

\begin{abstract}
:
The clothing industry is currently focused on $3 D$ virtual fitting. Many companies use size 12 as the core size; however, in recent years the average size has increased. For example, in the United Kingdom, the average size is now 16. Many companies have not updated their core size and often use size 12 as the size they are the most familiar with. The purpose of this paper is to compare real plus size body shapes with artificial avatars in relation to the fabric draping. This paper will investigate, how the body shape changes with an age (body height decreases, skin elasticity is lower, the shoulders are hunched, buttocks sag and fatness pockets are accumulated around the waist area). These factors are not considered in virtual avatars, but have a big impact on virtual fitting.
\end{abstract}

\section{Keywords:}

3D body scanning, body shapes, avatars, virtual fitting

\section{Introduction}

The apparel industry uses 3D virtual fitting for the development of clothing and has also started to expand the concept of $3 \mathrm{D}$ fitting by designing websites that allow customers to use a virtual avatar to determine, how an item of clothing will fit to their body.

Many research papers are currently focused on improving the fabric draping simulation by considering fabric properties, such as bending, stretching, weight, smoothness, etc. However, important factors such as the body firmness, body fat distribution, and fat pockets are not considered. This paper presents two problems that the apparel industry needs to consider [1]. The first issue is the changing body shape for plus sizes between ages $20-40$ and $40-60+$. In the younger age range, the body shape is more compact and firmer-breast tissue is firmer, there are less pockets of fat and the skin is tighter, which results in smoother body contours. In the older age range, the body shape undergoes many changes such as a sloping back, sagging breasts, and the stomach and buttocks lose skin elasticity causing them to droop [2]. The second issue is the body fat distribution. Virtual avatars that are currently used in 3D virtual fitting software do not accurately represent body imperfections such as lumps of fat that are formed in various locations around the body. Software developers, who are responsible for creating virtual avatars, should look to introduce these factors when designing the avatar. This will allow the customer to see a more accurate representation of how the clothing will fit to their body. Also, by improving the accuracy of the avatar body shape, pattern makers would benefit by being able to develop superior fitting clothing [3, 4].
A previous study shows a possible solution to improve the accuracy of virtual fitting by using control points. These are a series of points distributed around morphological contours that define the overall body shape (Figure 1).

By offsetting the control points outward from the gravity center of the surface of given contour, it is possible to define the shape of garment patterns. It has been shown that this method can improve the overall fit and allow better control of draping. The study focused specifically on the fitting of trousers. Figure 2 shows how this method of using control points can be used to form the trousers shape, defining the crotch line (Figure 2a), the outside and inside trousers lines (Figure 2b), and their surface of front and back (Figure 2c).

The human body is not perfectly symmetrical. When the body is scanned using a $3 \mathrm{D}$ scanner such as $\mathrm{TC}^{2}$, independent

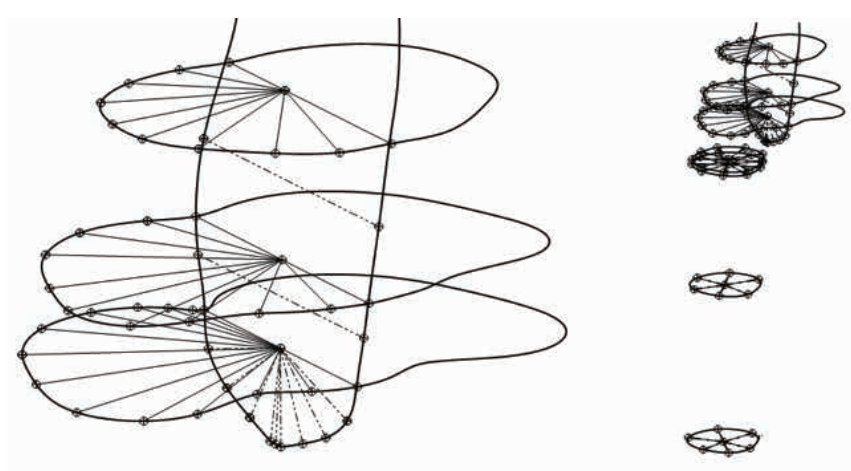

Figure 1. Distribution of the control points assigned to the morphological contours [4] 

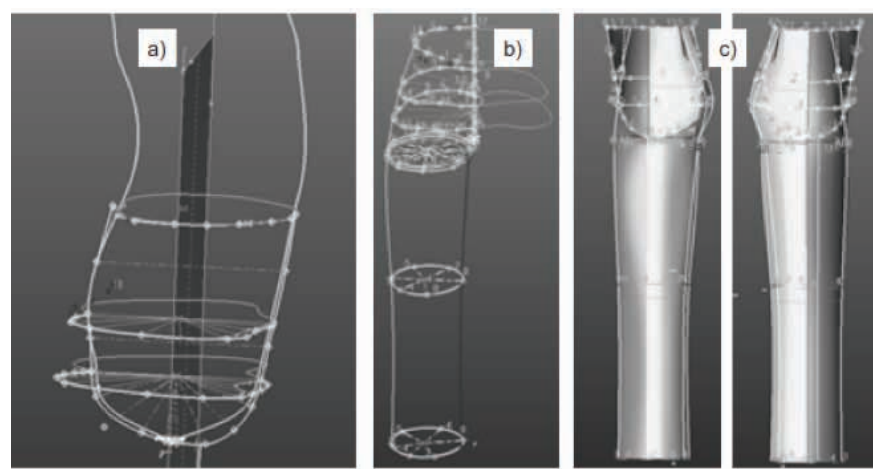

Figure 2. Numeric molding of trousers [4]

measurements can be obtained for the left and right side of the body. These data can be used to create very accurate patterns that are tailored to that person. When using $3 \mathrm{D}$ virtual fitting software, the avatar measurements can be adjusted, but the avatar is always symmetrical. Another issue with 3D virtual fitting software is that there are a finite number of key measurements that are used to define the overall body shape, and all other measurements are estimated from those. When the body is scanned using a 3D scanner, the entire body is digitally mapped; therefore, all measurements are accurate.

The aim of this paper is to highlight some limitations of the current virtual avatars. The contours of virtual avatars are much smoother than those found on the human body. Also, companies that develop virtual 3D fitting software such as Optitex, Browzer, and Lectra only allow the user to manipulate a limited number of body metrics. Due to these limitations, it is impossible to achieve the level accuracy that 3D body scanning captures.

\section{D body scan versus avatar}

For this research, a plus size woman (size 20) was scanned using a Size Stream scanner and 240 measurements were obtained. The person scanned was in the age range 40-60 and was wearing the underwear, when scanned.

The basic measurements were as follows: chest circumference- $116 \mathrm{~cm}$, waist circumference- $112 \mathrm{~cm}$, and hip circumference- $115 \mathrm{~cm}$. Figure 3 shows the real body shape with the real body fat distribution. Figure 4 shows a computergenerated image derived from the body scan data $[3,5,6]$.

The real 3D body scan images for the front, side, and back (Figure 3) were superimposed over the avatar front, side, and back images (Figure 3) to allow a direct comparison. Photoshop was used to change the color of the real 3D body scan images to green, so that there would be a contrast between them and the avatar. Illustrator was then used to overlay the real and avatar images to generate Figure 5 (see below). The reference point used to align the two images was the body right calf.

As can be seen from the comparison above (Figure 5), the real 3D body scan shows that the shoulders are less sloped, and the position and length of the shoulders are also different. In this case, if clothes patterns were developed using the
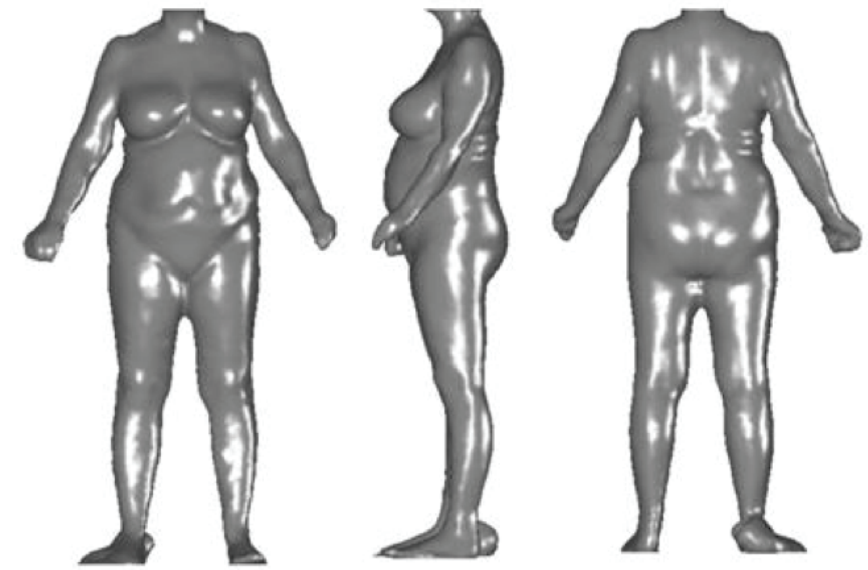

Figure 3. Real 3D body scan image

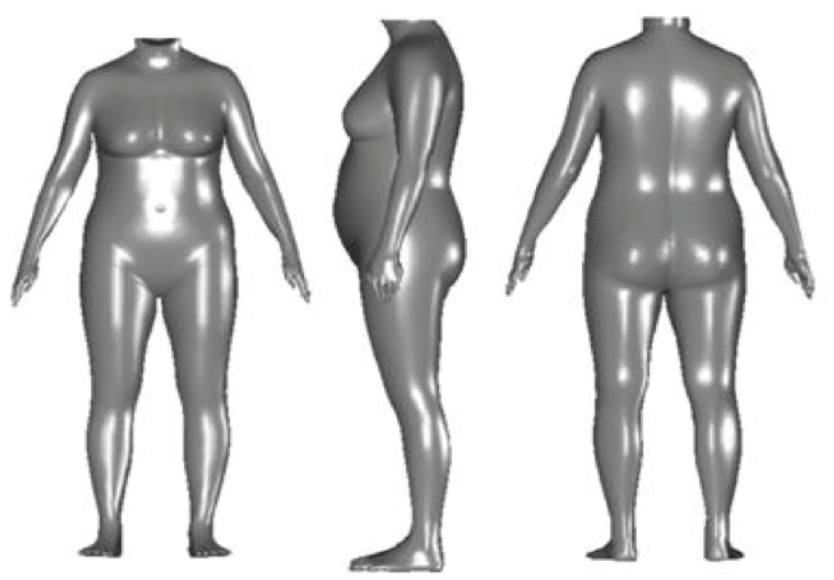

Figure 4. Avatar based on the body scan data
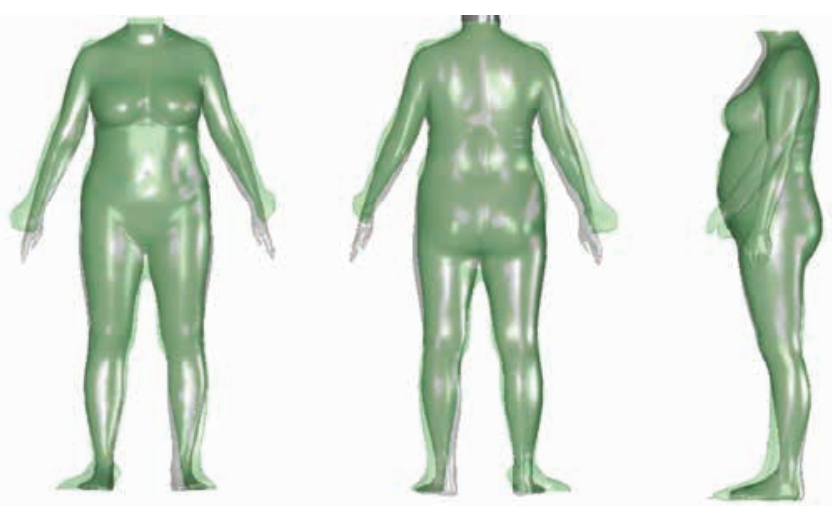

Figure 5. Real 3D body scan images (in green) shown superimposed over the corresponding avatar images (in grey)

avatar, the shoulder angle would be too steep. The biceps muscles are well defined on the real 3D body scan, where on the avatar the muscles are not defined resulting in straight contours. This could affect development of sports clothes as the sports clothing industry has recently started developing the compresion clothing, which controls the blood flow to the muscles. In this scenario, it is critical that the muscle contours are mapped to develop this type of clothing. The woman, who was scanned, was wearing a bra; however, it is still noticeable that the breasts are sagging. On the avatar, the breasts appear to be positioned higher and are firmer. Also, from the side view, it can be seen that the chest area protrudes more in the avatar, when compared to the real body scan. This could affect the 
across front measurements and also garment draping on the upper body. The 3D body scan reveals that there is a distribution of fat from under the bust line that extends down to the high hip area. The contours of the 3D body scan show lumps of fat situated on the flanks; it can also be seen that the skin has lost some elasticity. When comparing the fat distribution between $3 \mathrm{D}$ body scan and avatar, the fat on the avatar is accumulated only on the front of the body (exactly around the waist position); whereas on the 3D body scan it can be seen that there is more distributed directly under the breasts area [3, 6--10].

Body morphology of the virtual avatar (muscle layout and structure, skeletal system, fat distribution, etc.) is presented in references $[4,11,12]$. The authors thease papers created a virtual avatar by using data from a 3D scanner and then applied the cloth simulation to the avatar. Using the real scan data, a virtual avatar was created that preserved the specifics of the morphology of the scanned person, thus providing an accurate simulation of the clothing fitting, which is close to the real fit on the human body. Nevertheless, these works emphasize the need for individual processing of data from the scanner [11].

A further challenge faced by the apparel industry is creating garments for disabled people; for example, people with curvature of the spine, where the body shape is asymmetrical. Current 3D CAD/CAM software does not allow for asymmetric body shapes and it is therefore unsuitable for this application. In such a situation, the only viable solutions are either to manually measure the body or use real 3D body scanning to generate an accurate model of the body [12].

\section{CAD/CAM software and the avatar}

In this paper, the Lectra Modaris V7 software avatar was analyzed. Similar to the $\mathrm{TC}^{2}$ software, the avatar contours look too smooth, the body looks perfectly symmetrical, the fat is accumulated only on the front of the body, and there are no pockets of fat as can be seen on the real body (Figure 6). It is impossible to create an accurate avatar for somebody aged $60+$ as the skin structure and body firmness cannot be altered.
The Lectra software allows the user to define a set number of measurements, such as the height, silhouette, 14 torso measurements, 8 leg measurements, 4 arm measurements, and 6 morphology measurements (Figure 7) [7-9].

The Lectra software parameters do not allow the user to adjust the body fat distribution or body shape contours. This could affect the fitting of clothing, especially clothing that has a small ease [13]. Lectra software user wanting to create a virtually fitted garment with realistic cloth draping will encounter issues due to the avatar lacking real life imperfections, such as fat pockets, lack of body firmness, and asymmetry. These are important factors that affect cloth draping, especially for thin material. Another challenge facing CAD/CAM operators is that they are unable to update the fabric library. To simulate cloth draping for a new material, the software fabric library needs to be updated with the new fabric's parameters and weaving. It is not possible for the user to input these fabric properties by themselves. Any new fabric must be sent to the CAD/CAM software supplier to be tested and added to the library.
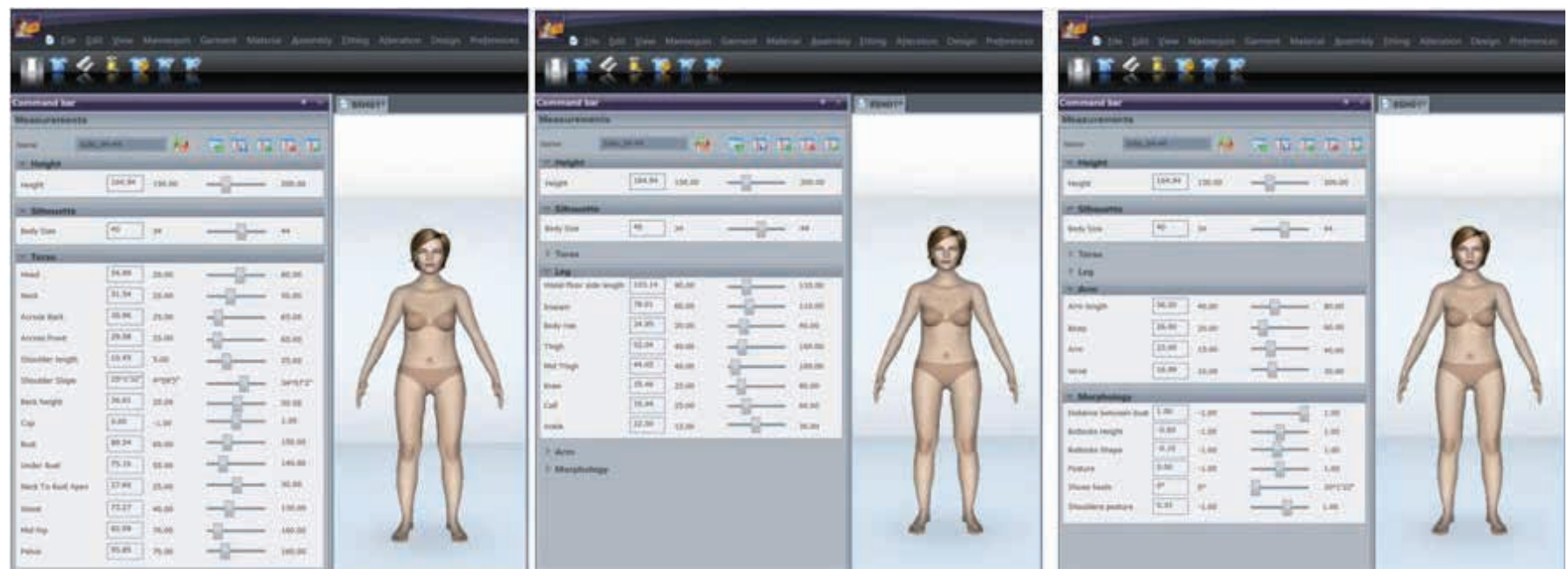

Figure 7. Lectra Modaris V7 body measurement parameters 


\section{Visual comparison of fabric draping on a real body and virtual avatar}

The image shown in Figure 8 presents the virtual avatar, which was created based on the real model. The patterns for the basic jersey dress were developed using the Gerber software and were virtually fitted using the Optitex software. The real dress was made using the same patterns as those used for the virtual dress. The fabric was chosen by the author and was a polycotton elastane ( $95 \%$ polyester, $5 \%$ elastane). The fabric was tested to determine its fabric parameters using FAST system. The fabric parameters are as follows: mass$216 \mathrm{~g} / \mathrm{m}^{2}$, friction coefficient-0.2, thickness-0.83 $\mathrm{mm}$, bending $-24.06 \mu \mathrm{Nm}$ and $23.26 \mu \mathrm{Nm}$, and shear $-33.01 \mathrm{~N} / \mathrm{m}$.

Comparing the virtual body shape with the real body shape (Figure 8), it can be seen that the virtual body posture it is straight; whereas on the real body the back is angled backwards and the bust is raised up. The shoulders slope down more on the virtual avatar than on the real body, which has more fat concentrated in this area. The virtual avatar has protruding buttocks, whereas the real body has flatter, sagging buttocks.

The aforementioned differences between the real body and virtual avatar body shapes have an effect on the fabric draping. On the real body, the back is angled backwards and the bust is raised, which affects the dress balance between the front and back (the dress is pulled to the back). The extra pockets of fat around the shoulders on the real body cause lots of gathering around the neck area that are absent on the virtual avatar, which looks perfectly smooth. The back of the dress on the real body is longer than on the virtual avatar. The dress is raised up at the back on the virtual model, because the fabric is suspended on the protruding buttocks, whereas Figure 9 shows that the fabric does not have as much support and so drapes lower. On the virtual avatar, the fold bending angles are much smaller than on the real body because they have been calculated incorrectly by the algorithm using fabric properties. The areas where the folds originate, are different due to a difference in fat distribution. The folds on the real dress, caused by gravitational forces, have formed from the waistline due to the fat concentrated above the high waistline. However, the fold are form below the waistline on the avatar, because the fat is concentrated below the waistline [14].
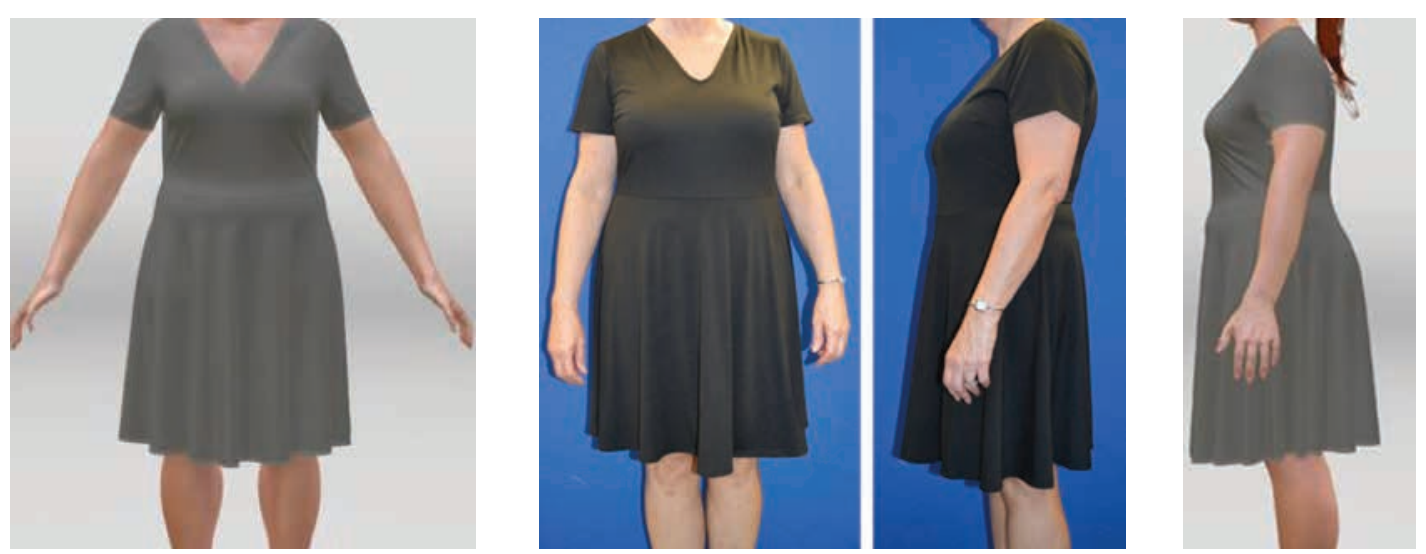

This paper has highlighted issues with current software used to generate plus size avatars. These programs do not accurately simulate the realistic fat distribution, real body asymmetry, skin elasticity, body firmness, and real muscle contours. The real body has many imperfections and is not perfectly symmetrical, resulting in the fabric bending angle being different from one side of the body to the other. Also, if the body morphology is uneven with lots of fatty lumps, the body fabric gap will be smaller, and the fabric may lay on the top of these lumps, which could affect the garment fitting and fabric draping. Virtual fitting is currently based on the avatar, which has perfectly smooth contours and is perfectly symmetrical. Therefore, it cannot simulate, how the fabric draping is affected by the asymmetry or lumpy body contours.

To improve the realism of avatars, CAD/CAM software companies should simulate body imperfections such as a lack of skin elasticity/body firmness, which causes the fabric to drape more and appear flatter on the body. From the reviewing literature, research shows that the body shape is affected by the lifestyle. The apparel industry has to keep an up-to-date measurement database and body shapes to ensure that garments fit correctly for the ever-changing population. Therefore, the CAD/CAM software user should have the ability to define the body fat distribution to ensure that the avatar represents the contemporary body shape.

A garment technologist, who does not understand the fabric behavior and who is reliant on virtual avatars to create clothing patterns, may encounter issues with clothes not fitting correctly due to the aforementioned software limitations. This could ultimately lead to a lack of customer satisfaction and loss of revenue.

\section{References}

[1] Streuber, S., Quiros-Ramirez, M. A., Hill, M. Q., Hahn, C. A., Zuffi, S., et al. (2016). Body talk: Crowdshaping realistic $3 D$ avatars with words. ACM Transactions on Graphics, 35, 54:1-54:14.

[2] Balach, M., Lesiakowska-Jablonska, M., Frydrych, I. (2019). Anthropometry and size groups in the clothing industry. Autex Research Journal. doi: 10.2478/aut-2019-0001.

Figure 8. Basic jersey dress_-virtual fitting (Optitex) and fitting on the real body 
[3] Henkel, R. Body scanners, simulations and avatars: The future is $3 D$. Retrieved April 25, 2019 from https://www. ispo.com/en/trends/id_79705556/body-scanners-andavatars-the-future-of-clothing-is-3d.html.

[4] Cichocka A., Bruniaux P., Frydrych I. (2016). 3D garment modelling-conception of its structure in $3 D$. Fibres \& Textiles in Eastern Europe, 24(4), 121-128.

[5] Fleming, R., Mohler, B. J., Romero, J., Black, M. J., Breidt, $M$. (2016). Appealing female avatars from $3 D$ body scans: Perceptual effects of stylization, 11th Joint Conference on Computer Vision, Imaging and Computer Graphics Theory and Applications, Vol. 1, February 27-29, Rome, Italy, pp. 335-345.

[6] Brownridge, A., Twigg, P. (2014). Body scanning for avatar production and animation. International Journal of Fashion Design, Technology and Education, 7(2), 125-132.

[7] Mattila, H. (2016). Digital fashion-How and when? 8th International Textile, Clothing and Design Conference, October 2-5, Dubrovnik, Croatia.

[8] Surville, J. M. (2010). Apparel: From reality to virtual reality, International Conference on $3 D$ Body Scanning Technologies, October 19-20, Lugano, Switzerland.
[9] D’Apuzzo, N. (2007). 3D body scanning technology for fashion and apparel industry. Electronic Imaging 2007, 6491, January 29, San Jose, CA, United States, pp. 1-12.

[10] Ballester, A., Parrilla, E., Uriel, J., Pierola, A., Alemany, S., et al. (2014). 3D-based resource fostering the analysis, use and exploitation of available body anthropometric data. 5th International Conference and Exhibition on $3 D$ Body Scanning Technologies, October 21-22, Lugano, Switzerland.

[11] Cichocka, A., Bruniaux P., Frydrych I. (2014). 3D garment modelling - creation of a virtual mannequin of the human body. Fibres \& Textiles in Eastern Europe, 22(6), 123-131.

[12] Bruniaux P., Cichocka A., Frydrych I. (2016). 3D digital methods of clothing creation for disabled people. Fibres \& Textiles in Eastern Europe, 24(5), 125-131.

[13] Magnenat-Thalmann, N., Kevelham, B., Volino, P., Kasap, M., Lyard, E. (2011). 3D Web-based virtual try on of physically simulated clothes. Computer-Aided Design and Applications, 8(2), 163-174.

[14] Yuan, M., Khan, I. R., Farbiz, F., Yao, S., Niswar, A., et al. (2013). A mixed reality virtual clothes try-on system. IEEE Transactions on Multimedia, 15(8), 1958-1968. 